\title{
QUASISTABLE GRADIENT AND HAMILTONIAN SYSTEMS WITH A PAIRWISE INTERACTION RANDOMLY PERTURBED BY WIENER PROCESSES
}

\author{
A. SKOROKHOD \\ Institute of Mathematics \\ Kiev, Ukraine \\ and \\ Michigan State University \\ Department of Statistics and Probability \\ East Lansing, MI 48824 USA
}

(Received February, 2002; Revised May, 2002)

Infinite systems of stochastic differential equations for randomly perturbed particle systems in $\boldsymbol{R}^{d}$ with pairwise interacting are considered. For gradient systems these equations are of the form

$$
d x_{k}(t)=F_{k}(t) d t+\sigma d w_{k}(t)
$$

and for Hamiltonian systems these equations are of the form

$$
d \dot{x}_{k}(t)=F_{k}(t) d t+\sigma d w_{k}(t) .
$$

Here $x_{k}(t)$ is the position of the $k$ th particle, $\dot{x}_{k}(t)$ is its velocity,

$$
F_{k}=-\sum_{j \neq k} U_{x}\left(x_{k}(t)-x_{j}(t)\right)
$$

where the function $U: R^{d} \rightarrow R$ is the potential of the system, $\sigma>0$ is a constant, $\left\{w_{k}(t), k=1,2, \ldots\right\}$ is a sequence of independent standard Wiener processes.

Let $\left\{x_{k}\right\}$ be a sequence of different points in $R^{d}$ with $\left|x_{k}\right| \rightarrow \infty$, and $\left\{v_{k}\right\}$ be a sequence in $R^{d}$. Let $\left\{\tilde{x}_{k}^{N}(t), k \leq N\right\}$ be the trajectories of the $N$-particles gradient system for which $\tilde{x}_{k}^{N}(0)=x_{k}, k \leq N$, and let $\left\{x_{k}(t), k \leq N\right\}$ be the trajectories of the $N$-particles Hamiltonian system for which $x_{k}^{N}(0)=x_{k}, \dot{x}_{k}(0)=v_{k}, k \leq N$. A system is called quasistable if for all integers $m$ the joint distribution of $\left\{x_{k}^{N}(t), k \leq m\right\}$ or $\left\{\tilde{x}_{k}^{N}(t), k \leq m\right\}$ has a limit as $N \rightarrow \infty$. We investigate conditions on the potential function and on the initial conditions under which a system possesses this property.

Key words: Configuration Space, Gradient and Hamiltonian Systems, Stochastic Differential Equations, Weak Convergence of Measures.

AMS subject classifications: 60H10, 60G46, 60K 35 .

\section{Introduction}

We consider a finite or an infinite sequence of $\boldsymbol{R}^{d}$-valued stochastic processes 
$x_{k}(t), k \in \mathcal{I}$

where $\mathcal{I}$ is a finite or infinite interval of the natural numbers by which the evolution of a set of pairwise interacting particles in the space $\boldsymbol{R}^{d}$ is described. The interaction is determined by a potential $\boldsymbol{U}(\boldsymbol{x})$ satisfying the condition

(P) $U(x)=u(|x|)$ where the function $u:(0,+\infty) \rightarrow R$ is smooth, $u(x)=0$ for $s>r$, and for some $\alpha<d$ the relation

$$
\lim _{s \downarrow 0} s^{\alpha+1} u^{\prime \prime}(s)=a
$$

is fulfilled, $r>0, a>0$ are constants.

The motion of a gradient system is determined by the system of the stochastic differential equations

$$
d x_{k}(t)=-\sum_{j \neq k} 1_{\{j \in \mathcal{I}\}} U_{x}\left(x_{k}(t)-x_{j}(t)\right) d t+\sigma d w_{k}(t), k \in \mathcal{I} .
$$

The number of equations coincides with the number of particles. $\boldsymbol{w}_{k}$ is the sequence of independent standard Wiener processes in $\boldsymbol{R}^{d}$. These equations are solved with some initial conditions $\left\{x_{k}(0)=x_{k}, k \in \mathcal{I}\right\}$, where $\left.\left\{x_{k}\right\}, k \in \mathcal{I}\right\}$, is a sequence of different points in $\boldsymbol{R}^{d}$, for which $\lim _{k}\left|x_{k}\right|=\infty$ if $\mathcal{I}$ is an infinite interval.

The motion of a Hamiltonian system is determined by the system of the stochastic differential equations

$$
d \dot{x}_{k}(t)=-\sum_{j \neq k} 1\{j \in \mathcal{I}\} U_{x}\left(x_{k}(t)-x_{j}(t)\right) d t+\sigma d w_{k}(t), k \in \mathcal{I}
$$

Here $\dot{x}_{k}(t)=\frac{d}{d t} x_{k}(t)$ is the velocity of the $k$ th particle. Equations (2) are solved with the initial conditions

$$
x_{k}(0)=x_{k}, \dot{x}_{k}(0)=v_{k},
$$

where $\left\{v_{k}\right\}, k \in \mathcal{I}$ is another sequence in $R^{d}$.

The existence of the solution to infinite systems of kind (1) was considered for smooth functions $\boldsymbol{U}(\boldsymbol{x})$ by J. Fritz (see [2]). Finite gradient and Hamiltonian systems with the potentials satisfying condition (P) were considered by the author (see [5]).

Quasistability. Let sequences $\left\{x_{k}, k \geq 1\right\}$ and $\left\{v_{k}, k \geq 1\right\}$ be given. For any $N>0$, there exists the solution to system (1) $\left\{x_{k}^{N}(t), k \leq N\right\}$, satisfying the initial conditions

$$
x_{k}^{N}(0)=x_{k}, k \leq N
$$

and the solution to system (2) $\left\{\widetilde{x}_{k}^{N}(T), k \leq N\right\}$ satisfying the initial conditions

$$
\widetilde{x}_{k}^{N}(0)=x_{k}, \frac{d}{d t} \widetilde{x}_{k}^{N}(0)=v_{k}, k \leq N
$$

This follows from the results of [5]. 
Gradient system (1) is called quasistable if for any $m$ the joint distribution of the stochastic processes $\left\{\widetilde{x}_{k}^{N}(\mathrm{t}), k \leq m\right\}$ has as a limit $N \rightarrow \infty$ in the space $\left(C_{[0, \infty)}\left(R^{d}\right)\right)^{m}$. Hamiltonian system (2) is called quasistable if for any $\boldsymbol{m}$ the joint distributions of the stochastic processes $\left\{\widetilde{x}_{k}^{N}(t), k \leq m\right\}$ has a limit $N \rightarrow \infty$ in the same space. Note that the quasistability is determined by the potential and initial conditions.

The main goal of this article is the investigation of the conditions on the potential and initial conditions of gradient and Hamiltonian systems under which they are quasistable.

\section{The Spaces $\Gamma$ and $\Gamma^{2}$}

It is convenient to consider gradient systems (1) in the configuration space $\boldsymbol{\Gamma}$ which is the set of locally finite counting measures $\gamma$ on the Borel $\sigma$-algebra $\mathcal{B}\left(R^{d}\right)$ of the space $R^{d}$. So, a measure $\gamma \in \Gamma$ satisfies the property:

$R^{d}$

(LF) the support $S_{\gamma}$ of the measure $\gamma$ is a sequence $\left\{x_{k}, k \geq 1\right\}$ of different points in

for which

$$
\sum_{k} 1_{\left\{\left|x_{k}\right|<n\right\}}<\infty
$$

for all $n>0$. The topology in $\Gamma$ is generated by the weak convergence of measures: $\gamma_{n} \rightarrow \gamma_{0}$ if

$$
\int \phi(x) \gamma_{n}(d x) \rightarrow \int \phi(x) \gamma_{0}(d x)
$$

for $\phi \in \mathcal{C}_{f}, \mathcal{C}_{f}$ is the set of continuous functions $\phi: R^{d} \rightarrow R$ with bounded support. Denote

$$
\langle\phi, \gamma\rangle=\int \phi(x) \gamma(d x), \phi \in \mathcal{C}_{f}
$$

and for a continuous function $\Phi:\left(R^{d}\right)^{2} \rightarrow R$ with bounded support

$$
\langle\Phi, \gamma \times \gamma\rangle=\int \Phi\left(x, x^{\prime}\right) \gamma(d x) \gamma\left(d x^{\prime}\right)-\int \Phi(x, x) \gamma(d x) .
$$

Using Ito's formula and considering the function $\boldsymbol{a}$ as a function of two variables: $\boldsymbol{a}\left(\boldsymbol{x}-\boldsymbol{x}^{\prime}\right)$, we can rewrite system (1) using $\Gamma$-valued function $\gamma_{t}$ for which

in the form

$$
\left\langle\phi, \gamma_{t}\right\rangle=\sum_{k} \phi\left(x_{k}(t)\right), \phi \in \mathcal{C}_{f}
$$

$d\left\langle\phi, \gamma_{t}\right\rangle=\left\langle\left\langle\phi^{\prime}, a\right), \gamma_{t} \times \gamma_{t}\right\rangle d t+\frac{\sigma^{2}}{2}\left\langle\Delta \phi, \gamma_{t}\right\rangle+\sum_{k} \sigma\left(\phi ;\left(x_{k}(t)\right), d w_{k}(t)\right)$

where

$$
\phi \in \mathcal{C}_{f}^{(2)}, \Delta \phi(x)=\operatorname{Tr} \phi^{\prime \prime}(x)
$$


and $\mathcal{C}_{f}^{(2)}$ is the set of those $\phi \in \mathcal{C}_{f}$ for which $\phi^{\prime}(x)$ and $\phi^{\prime \prime}(x)$ are continuous functions.

A $\Gamma$-valued stochastic process $\gamma_{t}(\omega)$ is called a weak solution to equation (4) if for all $\phi \in \mathcal{C}_{f}^{(2)}$ the stochastic process

$$
\mu_{\phi}(\omega, t)=\left\langle\phi, \gamma_{t}\right\rangle-\int_{0}^{t}\left[\left\langle\left(\phi^{\prime}, a\right), \gamma_{s} \times \gamma_{s}\right\rangle+\frac{\sigma^{2}}{2}\left\langle\Delta \phi, \gamma_{s}\right\rangle\right] d s
$$

is a martingale with respect to the filtration $\left(\mathcal{F}_{t}\right)_{t \geq 0}$ with the square characteristic

$$
\left\langle\mu_{\phi}, \mu_{\phi}\right\rangle_{t}=\sigma^{2} \int_{0}^{t}\left\langle\left(\phi^{\prime}, \phi^{\prime}\right), \gamma_{s}\right\rangle d s
$$

If $\gamma_{t}(\omega)$ is a weak solution to system (4) and

$$
\left\langle\phi, \gamma_{t}(\omega)\right\rangle=\sum_{k} \phi\left(x_{k}(t)\right)
$$

for $\phi \in \mathcal{C}_{f}$, then the sequence $\left\{x_{k}(t), k \in \mathcal{N}\right\}$ is a weak solution to system (1).

Compacts in $\Gamma$. For any $\gamma \in \Gamma$ and a continuous decreasing function

for which

$$
\lambda(t):(0, \infty) \rightarrow R_{+}
$$

$$
\lambda(0+)=+\infty, \lambda(+\infty)>0
$$

there exists a continuous decreasing function

such that

$$
\Phi(t):[0, \infty) \rightarrow R_{+}, \Phi(+\infty)=0
$$

$$
\iint \Phi(|x|) \Phi\left(\left|x^{\prime}\right|\right) \lambda\left(\left|x-x^{\prime}\right|\right) \gamma(d x) \gamma\left(d x^{\prime}\right)<\infty
$$

For any compact set $\boldsymbol{K}$ from $\boldsymbol{\Gamma}$ and any function $\boldsymbol{\lambda}$ satisfying the conditions mentioned before, there exists a function of the form given by relation (8) for which

$$
\sup _{\gamma \in K}\left\langle\Phi_{\lambda}, \gamma \times \gamma\right\rangle<\infty
$$

Note that the set

$$
\left\{\gamma:\left\langle\Phi_{\lambda}, \gamma \times \gamma\right\rangle \geq c\right\}
$$

is a compact in $\boldsymbol{\Gamma}$ for any $\boldsymbol{\Phi}_{\boldsymbol{\lambda}}$ of the form (8) and $\boldsymbol{c}>\mathbf{0}$. Denote by $\boldsymbol{\Gamma}_{\boldsymbol{\Phi}, \lambda}$ the set of those $\gamma \in \Gamma$ for which relation (7) is fulfilled. Set

$d_{\Phi, \lambda}\left(\gamma_{1}, \gamma_{2}\right)=$ 


$$
\sup \left\{\left|\left\langle\phi \Phi, \gamma_{1}\right\rangle-\left\langle\phi \Phi, \gamma_{2}\right\rangle\right|: \phi \in \operatorname{Lip}^{1} \mid\right\}+\left|\left\langle\Phi_{\lambda}, \gamma_{1} \times \gamma_{1}\right\rangle-\left\langle\Phi_{\lambda}, \gamma_{2} \times \gamma_{2}\right\rangle\right|
$$

where

$$
\operatorname{Lip}^{1}=\left\{\phi \in \mathcal{C}_{f}: \sup _{x}|\phi(x)| \leq 1, \sup _{x, x^{\prime}} \frac{\left|\phi(x)-\phi\left(x^{\prime}\right)\right|}{\left|x-x^{\prime}\right|} \leq 1\right\}
$$

$\Gamma_{\boldsymbol{\Phi}, \lambda}$ with the distance $\boldsymbol{d}_{\boldsymbol{\Phi}, \lambda}$ is a separable locally compact space.

It is convenient to consider Hamiltonian systems in the configuration space $\Gamma^{2}$ which is the subspace of those locally finite counting measures $\widehat{\gamma}$ in $\left(R^{d}\right)^{2}$ for which

$$
\sum_{k} \delta_{x_{k}} \in \Gamma,\left\{\left(x_{k} ; v_{k}\right), k>0\right\}=S_{\widehat{\gamma}}
$$

where $\left\{x_{k}, k>0\right\},\left\{v_{k}, k>0\right\}$ are sequences in the space $R^{d}$. Let $\left\{x_{k}(t), k>0\right\}$ be a solution to equation (2). Introduce a $\Gamma^{2}$-valued function

$$
\widehat{\gamma}(t, B)=\sum_{k} 1_{\left\{\left(x_{k}(t) ; \widehat{x}_{k}(t)\right) \in \mathcal{B}\right\}}, B \in \mathcal{B}\left(\left(R^{d}\right)^{2}\right)
$$

Let $\phi(x, v):\left(R^{d}\right)^{2} \rightarrow R$ be a function from $\mathcal{C}_{f}^{2}\left(\left(R^{d}\right)^{2}\right)$. Ito's formula implies the relations

$d\langle\phi, \widehat{\gamma}(t)\rangle=\left(\langle L \phi, \widehat{\gamma}(t)\rangle+\left\langle\left(\phi_{v}, a\right), \widehat{\gamma}(t) \times \widehat{\gamma}(t)\right\rangle\right) d t+\sigma \sum_{k}\left(\phi_{v}\left(x_{k}(t), \dot{x}_{k}(t)\right), d w_{k}\right.$ $(t))$

where

$$
L \phi(x, v)=\left(\phi_{x}(x, v), v\right)+\frac{1}{2} \sigma^{2} \operatorname{Tr} \phi_{v v}(x, v)
$$

and

$$
\left(\phi_{v}, a\right)=\left(\phi_{v}(x, v), a\left(x-x^{\prime}\right)\right), a(x)=-U_{x}(x)
$$

This means that the stochastic process $\widehat{\gamma}(t)$ is the solution to a martingale problem: for any $\phi \in \mathcal{C}\left(\left(R^{d}\right)^{2}\right)$ the stochastic process

$$
\widehat{\mu}_{\phi}(t, \omega)=\langle\phi, \widehat{\gamma}(t)\rangle-\int_{0}^{t}\left(\langle L \phi, \widehat{\gamma}(s)\rangle+\left\langle\left(\phi_{v}, a\right), \widehat{\gamma}(s) \times \widehat{\gamma}(s)\right\rangle\right) d s
$$

is a continuous martingale with the square characteristic

$$
\left\langle\widehat{\mu}_{\phi}\right\rangle_{t}=\int_{0}^{t}\left\langle\left|\phi_{v}\right|^{2}, \widehat{\gamma}(s)\right\rangle d s
$$

\section{Some Properties of Solutions to Finite Systems}


Assume that $d>2$ and the function $U(x)$ satisfies the condition $(\mathbf{P})$. In this section we consider solutions to systems (1) and (2) for which

$$
\mathcal{I}=\mathcal{I}_{n}=[1, n]
$$

Lemma 1:a) Let $\left\{x_{k}, k \leq n\right\}$ be different points in $\boldsymbol{R}^{d}$. Then the system of stochastic differential equations

$$
d \widetilde{x}_{k}(t)=\sum_{j \neq k} 1_{\{j \leq n\}} a\left(\tilde{x}_{k}(t)-\tilde{x}_{j}(t)\right) d t+\sigma d w(t),
$$

with initial value $\widetilde{x}_{k}(0)=x_{k}$ has a strong solution and this solution is unique.

b) Let $\left\{v_{k}, k \leq n\right\}$ be points in $\boldsymbol{R}^{d}$. Then system (2) with the initial conditions $x_{k}(0)=x_{k}, \dot{x}(0)=v_{k}, k \leq n$ has a strong solution and this solution is unique.

Proof: Set

$$
a^{\delta}(x)=-u^{\prime}(|x| \vee \delta) \frac{x}{|x|}, \delta>0
$$

Denote by $\left\{\widetilde{\boldsymbol{x}}_{k}^{\delta}(\boldsymbol{t}), \boldsymbol{k} \leq \boldsymbol{n}\right\}$ the solution to system (14) in which the function $\boldsymbol{a}(\boldsymbol{x})$ is changed to the function $\boldsymbol{a}^{\delta}(\boldsymbol{x})$, the existence and the uniqueness of this solution is a consequence of the Lipschitz condition of the function $\boldsymbol{a}^{\delta}(\boldsymbol{x})$. Let

$$
\delta_{0}=\inf \left\{\left|x_{k}-x_{j}\right|: k \leq n, j \leq n, k \neq j\right\} .
$$

Introduce the stopping times

$$
\tau_{\delta}=\inf \left\{t>0: \inf \left\{\left|\widetilde{x}_{k}^{\delta}(t)-\widetilde{x}_{k}^{\delta}(t)\right|: k \leq n, j \leq n, j \neq k\right\}, \delta>0 .\right.
$$

Then for $0<\delta_{2}<\delta_{1}<\delta_{0}$ we have

$$
\begin{gathered}
\tau_{\delta_{2}}>\tau_{\delta_{1}}, \\
\widetilde{x}_{k}^{\delta_{1}}(t)=\widetilde{x}_{k}^{\delta_{2}}(t), k \leq n, t \leq \tau_{\delta_{1}} .
\end{gathered}
$$

Let $h\left(x, x^{\prime}\right)=\left|x-x^{\prime}\right|^{2-d}$ and set

$$
z^{\delta}(t)=\sum_{k \neq j} 1_{\{k \leq n, j \leq n\}} h\left(\widetilde{x}_{k}^{\delta}(t), \widetilde{x}_{j}^{\delta}(t)\right) .
$$

Then

$$
d z^{\delta}(t)=\left(\sum_{i \neq j} S_{2}\left(\widetilde{x}_{k}^{\delta}(t), \widetilde{x}_{k}^{\delta}(t)\right)+\sum_{i \neq j \neq k \neq i} S_{3}\left(\widetilde{x}_{i}^{\delta}(t), \widetilde{x}_{j}^{\delta}(t), \widetilde{x}_{k}^{\delta}(t)\right)\right) d t+d \mu^{\delta}(t)(17)
$$

where

$$
\mathrm{S}_{2}^{\delta}\left(x_{1}, x_{2}\right)=-c_{2}\left|x_{1}-x_{2}\right|^{-3}\left(x_{1}-x_{2}, a^{\delta}\left(x_{1}-x_{2}\right)\right),
$$




$$
S_{3}^{\delta}\left(x_{1}, x_{2}, x_{3}\right)=-c_{3}\left|x_{1}-x_{2}\right|^{-d}\left(x_{1}-x_{2}, a^{\delta}\left(x_{1}-x_{3}\right)\right),
$$

$c_{2}, c_{3}$ are positive constants, and $\mu^{\delta}(t)$ is a martingale with the square characteristic

$$
\left\langle\mu^{\delta}\right\rangle_{t}=\int_{0}^{t} C\left(\widetilde{x}_{1}^{\delta}(s), \ldots, \tilde{x}_{n}^{\delta}(s)\right) d s
$$

and

$$
C\left(x_{1}, \ldots, x_{n}\right)=4(2-d)^{2} \sum_{k=1}^{n}\left|\sum_{i \neq k}\right| x_{k}-\left.\left.x_{i}\right|^{-d}\left(x_{k}-x_{i}\right)\right|^{2}
$$

Using formulas (18) and (19) we can prove that for some constant $L(d, n)$ which does not depend on $\delta$, the inequality holds

$$
\sum_{i \neq j} S_{2}^{\delta}\left(x_{i}, x_{j}\right)+\sum_{i \neq j \neq k \neq i} S_{3}^{\delta}\left(x_{i}, x_{j}, x_{k}\right) \leq L(d, n)
$$

for any set of different points

$$
\left\{\mathrm{x}_{k} \in R^{d}, k=1, \ldots, n\right\} .
$$

Formulas (14) and (17) imply that the stochastic process

$$
z^{\delta}(t)-L(d, n)(t-T)
$$

is a non-negative supermartingale on the interval $[0, T]$. So, for any $T>0$ we have the relation

$$
\lim _{c \rightarrow \infty} \sup _{\delta>0} P\left\{\sup _{t \leq T} z^{\delta}(t)>c\right\}=0 .
$$

This implies the relation

$$
P\left\{\lim _{\delta \rightarrow 0} \tau_{\delta}=+\infty\right\}=1
$$

Statement a) of the lemma is proved.

Let $\left\{x_{k}^{\delta}(t), k \leq n\right\}$ be the solution to the system of the stochastic differential equations

$$
d \dot{x}_{k}^{\delta}(t)=\sum_{j \neq k} 1\{j \leq n\} a^{\delta}\left(x_{k}^{\delta}(t)=x_{j}^{\delta}(t)\right) d t+\sigma d w_{k}(t), k \leq n
$$

with the initial conditions

$$
x_{k}^{\delta}(0)=x_{k}, \dot{x}_{k}^{\delta}(0)=v_{k}, k \leq n
$$


The existence and the uniqueness of the strong solution follows from the Lipschitz property of the function $a^{\delta}$. Let below the stopping time $\tau_{\delta}$ be determined by formula (16) with $x_{k}^{\delta}(t)$, $x_{j}^{\delta}(t)$ instead of $\widetilde{x}_{k}^{\delta}(t), \widetilde{x}_{j}^{\delta}(t)$. Set

$$
\begin{gathered}
U^{\delta}(x)=\int_{0}^{|x|} u^{\prime}(s \vee \delta) d s \\
Z_{\delta}(t)=\sum_{k \leq n}\left|\dot{x}_{k}^{\delta}(t)\right|^{2}+\sum_{k \leq n, j \leq n} U^{\delta}\left(x_{k}^{\delta}(t)-x_{j}^{\delta}(t)\right) .
\end{gathered}
$$

It is easy to calculate that

$$
d Z_{\delta}(t)=n \sigma^{2} d t+2 \sigma \sum_{k \leq n}\left(\dot{x}_{k}^{\delta}, d w_{k}(t)\right)
$$

So, the stochastic process

$$
Z_{\delta}(t)-n \sigma^{2}(t-T)-n u_{0}
$$

where $u_{0}=\inf _{s>0} u(s)$, is a non-negative martingale on the interval $[0, T]$ for all $\boldsymbol{T}>0$, $\delta>0$. Further proof of statement b) is the same as for statement a). The lemma is proved.

\section{Free Particles Processes}

We consider now the solution to equations (1) and (2) for $U(\cdot)=0$. They are called free particles processes. The stochastic process

$$
\tilde{X}_{n}^{*}(t)=\left\{x_{k}^{*}(t)=x_{k}+\sigma w_{k}(t), k \leq n\right\}
$$

in the space $\left(\boldsymbol{R}^{d}\right)^{n}$ is called an $\boldsymbol{n}$-particles free gradient process, and the stochastic process

$$
X_{n}^{*}(t)=\left\{x_{k}^{*}(t)=x_{k}+t v_{k}+\sigma \int_{0}^{t} w_{k}(s) d s, k \leq n\right\}
$$

in the same space is called an $\boldsymbol{n}$-particles free Hamiltonian process. We use the notations

$$
\tilde{X}_{n}(t)=\left\{\widetilde{x}_{k}(t), k \leq n\right\}, X_{n}(t)=\left\{x_{k}(t), k \leq n\right\}
$$

for the stochastic processes introduced in Lemma 1. Let $\tilde{\boldsymbol{m}}_{n}^{t}, \boldsymbol{m}_{n}^{t}, \tilde{\boldsymbol{m}}_{n}^{* t}$ be probability measures on the space $\left(\mathcal{C}_{[0, t]}\left(R^{d}\right)\right)^{n}$ which are the distributions of the stochastic processes

$$
\tilde{X}_{n}(\cdot), X_{n}(\cdot), \tilde{X}_{n}^{*}(\cdot), X_{n}^{*}(\cdot)
$$

on the interval $[0, t]$.

Lemma 2: a) The measure $\tilde{\boldsymbol{m}}_{n}^{t}$ is absolutely continuous with respect to the measure $\tilde{\boldsymbol{m}}_{n}^{* t}$, and the measure $\boldsymbol{m}_{n}^{t}$ is absolutely continuous with respect to the measure $\boldsymbol{m}_{n}^{* t}$ for all $\boldsymbol{t}>\mathbf{0}$.

b) Denote

$$
Z_{n}(\cdot)=\left(z_{1}(\cdot) ; \ldots ; z_{n}(\cdot)\right), z_{k}(\cdot) \in \mathcal{C}_{] 0, \infty)}\left(R^{d}\right), k \leq n
$$

and 


$$
\begin{aligned}
& \frac{d \tilde{m}_{n}^{t}}{d \tilde{m}^{* t}}\left(Z_{n}(\cdot)\right)=\tilde{d}_{n}\left(t, Z_{n}(\cdot)\right) \\
& \frac{d m_{n}^{t}}{d m_{n}^{* t}}\left(Z_{n}(\cdot)\right)=d_{n}\left(t, Z_{n}(\cdot)\right)
\end{aligned}
$$

Then

$$
\begin{gathered}
\tilde{d}_{n}\left(t, \tilde{X}_{n}^{*}(\cdot)=\tilde{\rho}(t)\right) \\
\exp \left\{\frac{1}{\sigma} \sum_{k \leq n} \int_{0}^{t} \tilde{A}_{n}\left(s, \tilde{x}_{k}^{*}(s) d w_{k}(s)-\frac{1}{2 \sigma^{2}} \int_{0}^{t} \sum_{k \leq n}\left|\tilde{A}_{n}\left(s, \widetilde{x}_{k}^{*}(s)\right)\right|^{2} d s\right\}\right.
\end{gathered}
$$

where

$$
\tilde{A}_{n}(s, x)=\sum_{k \leq n} 1_{\left\{\tilde{x}_{k}^{*}(s) \neq x\right\}} a\left(x-\tilde{x}_{k}^{*}(s)\right),
$$

and

$$
\begin{gathered}
d_{n}\left(t, X_{n}^{*}(\cdot)=\rho(t)\right) \\
\exp \left\{\frac{1}{\sigma} \sum_{k \leq n} \int_{0}^{t} A_{n}\left(s, x_{k}^{*}(s) d w_{k}(s)-\frac{1}{2 \sigma^{2}} \int_{0}^{t} \sum_{k \leq n}\left|A_{n}\left(s, x_{k}^{*}(s)\right)\right|^{2} d s\right\}\right.
\end{gathered}
$$

where

$$
A_{n}(s, x)=\sum_{k \leq n} 1_{\left\{x_{k}^{*}(s) \neq x\right\}} a\left(x-x_{k}^{*}(s)\right)
$$

Proof: Denote by $\widehat{\boldsymbol{m}}_{n}^{\delta, t}$ the distribution of the stochastic process

$$
\tilde{X}_{n}^{\delta}(\cdot)=\left\{\tilde{x}_{k}^{\delta}(\cdot), k \leq n\right\}
$$

in the space $\left(\mathcal{C}_{[0, t]}\left(R^{d}\right)\right)^{n}$. This stochastic process was introduced in the proof of Lemma 1. It follows from the results of [5] that $\tilde{\boldsymbol{m}}_{n}^{t} \ll \tilde{\boldsymbol{m}}_{n}^{* t}$ and

$$
\begin{gathered}
\tilde{\rho}_{n}^{\delta}(t)=\frac{d \tilde{m}_{b}^{\delta, t}}{d \tilde{m}_{n}^{* t}}\left(\tilde{X}^{*}(\cdot)\right) \\
=\exp \left\{\sum_{k \leq n} \frac{1}{\sigma} \int_{0}^{t} \tilde{A}_{n}^{\delta}\left(s, \tilde{x}_{k}^{*}(s) d w_{k}(s)-\frac{1}{2 \sigma^{2}} \int_{0}^{t} \sum_{k \leq n}\left|\tilde{A}_{n}^{\delta}\left(s, \tilde{x}_{k}^{*}(s)\right)\right|^{2} d s\right\}\right.
\end{gathered}
$$

where $\tilde{A}_{n}^{\delta}(s, x)$ is calculated by formula (22) with $\boldsymbol{a}^{\delta}$ instead of $\boldsymbol{a}$. It is easy to see that

$$
\tilde{X}_{n}^{\delta}(t)=\tilde{X}_{n}(t), \tilde{\rho}_{n}^{\delta}(t)=\tilde{\rho}_{n}(t)
$$

for $\boldsymbol{t} \leq \tau_{\delta}$ (see formula (16)). This implies formula (21). Formula (23) is proved in the same way. The lemma is proved.

\section{Infinite Free Particles System}


Let $\left\{x_{k}, k \leq 1\right\}$ be a sequence in $R^{d}$ for which $\gamma=\sum_{k} \delta_{x_{k}} \in \Gamma$, and let $\left\{v_{k}, k \geq 1\right\}$ be an arbitrary sequence in $\boldsymbol{R}^{d}$. We consider these two sequences of stochastic processes:

$$
\left\{\tilde{x}_{k}^{*}(t)=x_{k}+\sigma w_{k}(t), k \geq 1\right\},\left\{x_{k}^{*}(t)=x_{k}+t v_{k}+\sigma \int_{0}^{t} w_{k}(s) d s, k \leq 1\right\}
$$

Lemma 3: a) The relation

$$
P\left\{\sum_{k} 1_{\left\{\left|\tilde{x}_{k}^{*}(t)\right|<c\right\}}<\infty\right\}=1
$$

is fulfilled for all $\boldsymbol{t}>\mathbf{0 ,} \boldsymbol{c}>\mathbf{0}$ if $\boldsymbol{\gamma}$ satisfies condition

(EGFPS) (existence of gradient free particle system) for all $\boldsymbol{t}>\mathbf{0}$

$$
\int \exp \left\{-t|x|^{2}\right\} \gamma(d x)<\infty .
$$

b) The relation

$$
P\left\{\sum_{k} 1_{\left\{\left|x_{k}^{*}(t)\right|<c\right\}}<\infty\right\}=1
$$

is fulfilled for all $\boldsymbol{t}>\mathbf{0}, \boldsymbol{c}>\mathbf{0}$ if the sequences $\left\{\boldsymbol{x}_{\boldsymbol{k}}\right\},\left\{\boldsymbol{v}_{\boldsymbol{k}}\right\}$ satisfy the condition

(EHFPS) (existence of Hamiltonian free particle system) for all $\boldsymbol{t}>\mathbf{0}, \boldsymbol{s}>\mathbf{0}$

$$
\sum_{k} \exp \left\{-\boldsymbol{t}\left|\boldsymbol{x}_{k}-\boldsymbol{s} \boldsymbol{v}_{k}\right|^{2}\right\}<\infty
$$

Proof: It follows from Kolmogorov's theorem on the convergence of random series that relation (26) is equivalent to the relation

$$
\sum_{k} P\left\{\left|x_{k}^{*}(t)\right|<c\right\}<\infty
$$

Using the inequality

$$
\left|\left(x, x_{k}\right)\right| \leq \frac{1}{4}\left|x_{k}\right|^{2}+|x|^{2}
$$

we can prove the existence of positive continuous functions

$$
A(t, c), B(t, c), t>0, c>0,
$$

for which the inequalities below are held

$$
A(c, t) \exp \left\{-\frac{1}{\sigma^{2} t}\left|x_{k}\right|^{2}\right\} \leq P\left\{\left|x_{k}^{*}(t)\right|<c\right\} \leq B(c, t) \exp \left\{-\frac{1}{4 \sigma^{2} t}\left|x_{k}\right|^{2}\right\} .
$$

This implies statement a). Statement b) can be proved in the same way. The lemma is proved.

Corollary 1: If condition (EGFPS) is fulfilled, then the relations 


$$
\left\langle\phi, \tilde{\gamma}_{t}^{*}(\gamma, \omega)\right\rangle=\sum_{k} \phi\left(x_{k}^{*}(t), \phi \in \mathcal{C}_{f}\right.
$$

determine a $\Gamma$-valued continuous stochastic process $\widetilde{\gamma}_{t}^{*}(\gamma, \omega)$ which is called a gradient free particles process.

If condition (EHFPS) is fulfilled, then the relations

$$
\left\langle\phi, \gamma_{t}^{*}(\gamma, \omega)\right\rangle=\sum_{k} \phi\left(x_{k}^{*}(t)\right), \phi \in \mathcal{C}_{f}
$$

where $\widehat{\gamma}=\sum_{k} \delta_{\left(x_{k} ; v_{k}\right)} \in \Gamma^{2}$ determine a $\Gamma$-valued stochastic process $\gamma_{t}^{*}(t, \omega)$ which is called $a$ Hamiltonian free particle process.

Remark 1: Let $\left\{x_{k}\right\}$ satisfy condition (EGFPS), and

$$
\left|v_{k}\right|=o\left(\left|x_{k}\right|\right), k \rightarrow \infty
$$

Then condition (EHFPS) is fulfilled.

Introduce for $1 \leq m<n$ the stopping times with respect to the filtration $\left\{\mathcal{F}_{t}, t \geq 0\right\}$ generated by the sequence $\left\{w_{k}(t)\right\}$ :

$$
\begin{aligned}
& \tilde{\zeta}_{m, n}=\inf \left\{t>0: \min \left\{\left|\tilde{x}_{t}^{*}(t)-\tilde{x}_{t}^{*}(t)\right|: i \leq m, k \geq n\right\} \leq r\right\} \\
& \zeta_{m, n}=\inf \left\{t>0: \min \left\{\left|x_{t}^{*}(t)-x_{t}^{*}(t)\right|: i \leq m, k \geq n\right\} \leq r\right\}
\end{aligned}
$$

Lemma 4: a) Let condition (EGFPS) be fulfilled. Then for any $\boldsymbol{t}>\mathbf{0}, \boldsymbol{m} \geq \mathbf{1}$ the relation

$$
\lim _{n} P\left\{\widetilde{\zeta}_{m, n} \leq t\right\}=0
$$

is held.

b) Let condition (EHFPS) be fulfilled. Then for any $\boldsymbol{t}>0, m \geq 1$ the relation

$$
\lim _{n} P\left\{\zeta_{m, n} \leq t\right\}=0
$$

is held.

Proof: Let us prove statement a). It suffices to prove the relation

$$
\lim _{n} P\left\{\inf _{s \leq t} \inf _{i>n}\left|\widetilde{x}_{i}^{*}(s)\right| \leq c\right\}=0
$$

for any $t>0, c>0$. This follows from the inequality

$$
P\left\{\inf _{s \leq t} \inf _{i>n}\left|\widetilde{x}_{i}^{*}(s)\right| \leq c\right\} \leq \sum_{i>n} P\left\{\inf _{s \leq t}\left|\widetilde{x}_{k}^{*}(s)\right| \leq c\right\} .
$$

The lemma is proved. 


\section{Quasistability}

First, we calculate the conditional expectation $\boldsymbol{E}\left(\widetilde{\rho}_{n}(t) / \mathcal{F}_{t}^{m}\right)$ where the filtration $\left\{\mathcal{F}_{t}^{m}, t \geq 0\right\}$ is generated by the Wiener processes $\left\{\boldsymbol{w}_{k}(t), k \leq m\right\}$. We use the notation

$$
\tilde{\mu}_{n}(t)=\int_{0}^{t} \sum_{i \neq j} 1_{\{i \vee j \leq n\}}\left(a\left(\widetilde{x}_{i}^{*}(s)-\widetilde{x}_{j}^{*}(s)\right), d w_{i}(s)\right) .
$$

To simplify writing, we assume that $\sigma=\mathbf{1}$ and $\boldsymbol{r}=\mathbf{1}$. It follows from formula (21) that

$$
\widetilde{\rho}_{n}(t)=\exp \left\{\tilde{\mu}_{n}(t)-\frac{1}{2}\left\langle\widetilde{\mu}_{n}\right\rangle_{t}\right\}
$$

where $\left\langle\widetilde{\mu}_{n}\right\rangle_{t}$ is the square characteristic of the martingale $\widetilde{\mu}_{n}$. This implies the representation

$$
\tilde{\rho}_{n}(t)=1+\sum_{k=1}^{\infty} \int_{0<s_{1}<\ldots<s_{k}<t} d \tilde{\mu}_{n}\left(s_{1}\right) \ldots d \tilde{\mu}_{n}\left(s_{k}\right)
$$

Remark 2: Let

$$
\mu_{n}(t)=\int \sum_{i \neq j} 1_{\{1 \vee j \leq n\}}\left(a\left(x_{i}^{*}(s)-x_{j}^{*}(s)\right), d w_{i}(s)\right) .
$$

Then the density $\rho_{n}(t)$ (see formula (23)) is represented by relation (31) with $\mu_{n}$ instead $\tilde{\mu}_{n}$.

Lemma 5: Let $\boldsymbol{m}<\boldsymbol{n}$. Then

$$
\begin{gathered}
\tilde{\rho}_{m, n}(t)=E\left(\tilde{\rho}_{n}(t) / \mathcal{F}_{t}^{m}\right) \\
\exp \left\{\sum_{k \leq m} \int_{0}^{t}\left(b_{m, n}^{k}(s), d w_{k}(s)\right)-\frac{1}{2} \int_{0}^{t}\left|b_{m, n}^{k}(s)\right|^{2} d s\right\}
\end{gathered}
$$

and for $\boldsymbol{k} \leq \boldsymbol{m}$, the functions $\boldsymbol{b}_{m, n}^{k}(\boldsymbol{t})$ are determined by the formulas

$$
b_{m, n}^{k}(t)=\sum_{N \geq 1} \int \ldots \int_{t_{1}<\ldots<t_{N}<t} B_{m, n}^{k}\left(N, t_{1}, \ldots, t_{N}\right) d t_{1} \ldots d t_{N}
$$

where

$$
\begin{gathered}
B_{m, n}^{k}\left(N, t_{1}, \ldots, t_{N}\right) \\
=E\left(\sum_{i_{1}, j_{1}} 1_{(I J)_{N}^{k}}\left(\prod_{l<N}\left(a\left(\tilde{x}_{i_{1}}^{*}\left(t_{l}\right)-\tilde{x}_{j_{1}}^{*}\left(t_{l}\right)\right), \frac{w_{i_{1}}\left(t_{\kappa(l)}\right)-w_{i_{1}}\left(t_{l}\right)}{t_{\kappa(l)}-t_{l}}\right)\right)\right. \\
\left.a\left(\tilde{x}_{k}^{*}\left(t_{N}\right)-\tilde{x}_{j N}^{*}\left(t_{N}\right)\right) / \mathcal{F}_{t}^{m}\right),
\end{gathered}
$$

and $\kappa(l)=\inf \left\{l^{\prime}>l: i_{l} \in\left\{i_{l^{\prime}} ; j_{l^{\prime}}\right\}\right\}$ 


$$
\begin{gathered}
(I J)_{N, n}^{k}=\left\{\left(\left(i_{1} ; \ldots ; i_{n}\right) ;\left(j_{1}, \ldots ; j_{n}\right)\right) \in[1, n]^{N} \times[1, n]^{N}:\right. \\
\left.i_{l} \in[m+1, n] \cap\left\{j_{l+1}, \ldots, j_{n}\right\}, l<n, i_{N}=k, j_{l} \leq n, l<N, j_{N} \in[m+1, n]\right\}
\end{gathered}
$$

Proof: First note that the right-hand side of formula (32) gives the general representation for exponential martingales with respect to the filtration $\left\{\mathcal{F}_{t}^{m}, t \geq 0\right\}$ with the expectation 1 If the functions $b_{m, n}^{k}(t), k=1, \ldots m$ satisfy formula (32), then we can write the relation

$$
\begin{gathered}
\sum_{N \geq 1} \int \ldots \int_{0<t_{1}<\ldots<t_{N}<t} \sum_{k \leq m}\left(b_{m, n}^{k}\left(t_{1}\right), d w_{k}\left(t_{1}\right)\right) \ldots \sum_{k \leq m}\left(b_{m, n}^{k}\left(t_{N}\right), d w_{k}\left(t_{N}\right)\right. \\
=E\left(\sum_{N \geq 1} \int \ldots \int_{0<t_{1}<\ldots<t_{N}<t} d \tilde{\mu}_{n}\left(t_{1}\right) \ldots d \tilde{\mu}_{n}\left(t_{N}\right) / \mathcal{F}_{t}^{m}\right) \\
=E\left(\sum_{N \geq 1} \int_{0<t_{1}<\ldots<t_{N}<t} \sum_{j_{l} \neq i_{l}} 1_{\left\{i_{1} \vee j_{1} \leq n\right\}} \prod_{l \leq N}\left(a\left(\widetilde{x}_{i_{l}}^{*}\left(t_{l}\right)-\widetilde{x}_{j_{l}}^{*}\left(t_{l}\right)\right), d w_{i_{l}}\left(t_{l}\right)\right) / \mathcal{F}_{t}^{m}\right) .
\end{gathered}
$$

To determine the function $b_{m, n}^{k}(t)$, we have to collect all the terms in the right-hand side of the last equality representing the integral

$$
\int_{0}^{t}\left(b_{m, n}^{k}(s), d w_{k}(s)\right)
$$

It is easy to see these terms correspond to the sequences $\left\{i_{l}, l \leq N\right\}$ and $\left\{j_{l}, l \leq N\right\}$ which satisfy the conditions

$$
\left(\left(i_{1} ; \ldots ; i_{n}\right) ;\left(j_{1} ; \ldots ; j_{N}\right)\right) \in(I J)_{N, n}^{k} .
$$

Let $\boldsymbol{w}(\cdot)$ be a standard Wiener process in $\boldsymbol{R}^{d}$, and $\boldsymbol{s}<\boldsymbol{u}<\boldsymbol{t}$. Then the formula for conditional expectations is valid

$$
E(w(u)-w(s) / w(t))=\frac{w(t)-w(s)}{t-s}(u-s) .
$$

Here $\boldsymbol{E}(\cdot / \boldsymbol{w}(t))$ is the conditional expectation with respect to give $\boldsymbol{w}(t)$. This relation and description of $\boldsymbol{b}_{m, n}^{k}$ imply formulas (33), (34). The lemma is proved.

Let the sequences $\left\{i_{l}, l \leq N\right\}$ and $\left\{j_{l}, l \leq N\right\}$ satisfying the condition

$$
\left(\left(i_{1} ; \ldots ; i_{N}\right) ;\left(j_{1} ; \ldots j_{N}\right)\right) \in(I J)^{k_{N, n}}
$$

be fixed. Introduce notations

$$
\mathcal{J}=\left\{j_{l}: j_{l}>m\right\}, \mathcal{F}=\left\{j_{l}: j_{l} \leq m\right\}, \mathcal{I}=\left\{i_{1}, \ldots, i_{N}\right\}
$$

Denote

$$
\begin{gathered}
B_{m, n}^{k}\left(N ; i_{1}, \ldots, i_{N} ; j_{1}, \ldots, j_{n} ; t_{1}, \ldots, t_{N}\right) \\
=E^{*}\left(\left(\prod_{l<N}\left(a\left(\widetilde{x}_{i_{l}}^{*}\left(t_{l}\right)-\widetilde{x}_{j_{l}}^{*}\left(t_{l}\right)\right), \frac{w_{i_{l}}\left(t_{\kappa(l)}\right)-w_{i_{l}}\left(t_{l}\right)}{t_{\kappa(l)}-t_{l}}\right) a\left(\widetilde{x}_{k}^{*}\left(t_{N}\right)-\widetilde{x}_{j_{N}}^{*}(t)\right) / \mathcal{F}_{t}^{m}\right)\right.
\end{gathered}
$$


Here $\boldsymbol{E}^{*}$ is the expectation with respect to the joint distribution of the Wiener processes $\left\{w_{i}(\cdot), i \in \mathcal{J}\right\}$. We will use some graphs in the set $X(\mathcal{J})=\left\{x_{j_{l}}: j_{l} \in \mathcal{J}\right\}$.

Denote by $\boldsymbol{G}(\mathcal{J})$ the set of all connected graphs with the set of vertices

$$
V(G)=X(\mathcal{J})
$$

and the set of edges

$$
E(G) \subset\left\{\left\{x_{i_{l}}, x_{j_{l}}\right\}: i_{l}, l_{l} \in \mathcal{J}\right\} .
$$

A graph $G^{\prime} \in G(\mathcal{J})$ is called minimal if

$$
\operatorname{card} E\left(G^{\prime}\right)=\operatorname{card} \mathcal{J}-1 .
$$

The set of all minimal graphs is denoted by $M G(\mathcal{J})$.

Lemma 6: There exist some constants $c>0, \boldsymbol{\theta}>0$, and a graph $G^{\prime} \in M G(\mathcal{J})$ for which the inequality is valid

$$
\begin{gathered}
\left|B_{m, n}^{k}\left(N ; i_{1}, \ldots, i_{N} ; j_{1}, \ldots, j_{N} ; t_{1}, \ldots, t_{N}\right)\right| \leq c^{N} \Pi_{N}\left(t_{1}, \ldots, t_{N}\right) \\
\exp \left\{-\left(\sum \frac{\theta}{t_{l}}\left(\left|x_{i_{l}}-x_{j_{l}}\right|^{2}-1\right) \vee 01_{\left\{\left\{i_{l}, j_{l}\right\} \in E\left(G^{\prime}\right)\right\}}+\frac{\theta}{t_{N}}\left(\left|W_{k}\left(t_{N}\right)\right|^{2}\left(\widetilde{E}_{2}\left(G^{\prime}\right)\right)^{\frac{1}{2}}\right.\right.\right.
\end{gathered}
$$

where

$$
\begin{gathered}
\prod_{N}\left(t_{1}, \ldots, t_{N}\right)=\left(\prod_{1 \leq N}\left(t_{l}-t_{\iota(l)}\right)^{\alpha}\left(t_{\kappa(l)}-t_{l}\right)^{\left.1_{\{l<N\}}\right)^{-(1 / 2)}}\right. \\
\iota(l)=\max \left\{l^{\prime}<l: \operatorname{Card}\left(\left\{i_{l^{\prime}} ; j_{l^{\prime}}\right\} \bigcap\left\{i_{l} ; j_{l}\right\}\right)>0\right\} \\
\tilde{E}_{2}\left(G^{\prime}\right)=E^{*} \prod_{l \in \Lambda^{\prime}\left(G^{\prime}\right)} 1_{\left\{\left|\tilde{x}_{i l}^{*}\left(t_{l}\right)-\tilde{x}_{j_{l}}^{*}\left(t_{l}\right)\right| \leq 1\right\}}, \\
\Lambda^{\prime}\left(G^{\prime}\right)=\left\{l<N:\left\{x_{i_{l}}, x_{j_{l}}\right\} \notin E\left(G^{\prime}\right)\right\}
\end{gathered}
$$

Proof: Let

$$
p \in\left(1, \frac{d}{\alpha}\right), q_{1}>1, q_{2}>1, \frac{1}{p}+\frac{1}{q_{1}}+\frac{1}{q_{2}}=1 .
$$

We can write the inequality

$$
\begin{gathered}
\left|B_{m, n}^{k}\left(N ; i_{1}, \ldots, i_{N} ; j_{1}, \ldots, j_{N} ; t_{1}, \ldots, t_{N}\right)\right| \\
\leq\left(E^{*}\left(\prod_{l \leq N}\left|a\left(w_{i_{l}}\left(t_{l}\right)-w_{j_{l}}\left(t_{l}\right)+z_{l}\right)\right|^{p}\right)^{1 / p}\left(E^{*}\left(\prod_{l<N} \frac{\left|w_{i_{l}}\left(t_{\kappa(l)}\right)-w_{i_{l}}\left(t_{l}\right)\right|}{\sqrt{t_{\kappa(l)}-t_{l}}}\right)^{q_{1}}\right)^{1 / q_{1}}\right. \\
\left(E^{*}\left(\prod_{l \leq N} 1_{\left\{\left|w_{i_{l}}\left(t_{l}\right)-1_{\left\{j_{l}>m\right\}} w_{j_{l}}\left(t_{l}\right)+z_{l}\right| \leq 1\right\}}\right)^{q_{2}}\right)^{1 / q_{2}} \prod_{N}^{1}\left(t_{1}, \ldots, t_{N}\right),
\end{gathered}
$$


where

$$
\Pi_{N}^{1}\left(t_{1}, \ldots, t_{N}\right)=\prod_{l<N}\left(t_{\kappa(l)}-t_{l}\right)^{-1 / 2}
$$

It is easy to see that for some $c_{1}>0$, we can write the inequality

$$
E \mid a\left(w_{1}(t)+z\right)^{p}<c_{1} t^{-p \alpha / 2}, z \in R^{d}, t>0 .
$$

So

$$
E\left(\left|a\left(z_{l}-w_{i_{l}}\left(t_{l}\right)\right)\right|^{p} / \mathcal{F}_{t_{\iota(l)}}\right) \leq c_{1}\left(t_{l}-t_{\iota(l)}\right)^{-p \alpha / 2},
$$

and

$$
\left(E^{*}\left(\prod_{l \leq N}\left|a\left(w_{i_{l}}\left(t_{l}\right)-w_{j_{l}}\left(t_{l}\right)+z_{l}\right)\right|\right)^{p}\right)^{1 / p} \leq\left(c_{1}\right)^{N} \Pi_{N}^{2}\left(t_{1}, \ldots, t_{N}\right)
$$

where

$$
\Pi_{N}^{2}\left(t_{1}, \ldots, t_{N}\right)=\prod_{l \leq N}\left(t_{l}-t_{\iota(l)}\right)^{-\alpha / 2} .
$$

In addition, for some constant $c_{2}>0$ we can write the inequality

$$
E^{*}\left(\prod_{l \leq N} \frac{\left|w_{i_{l}}\left(t_{\kappa(l)}\right)-w_{i_{l}}\left(t_{l}\right)\right|}{\sqrt{t_{\kappa(l)}-t_{l}}}\right)^{q_{1}} \leq c_{2}^{N}
$$

Now we proceed to estimation of the expectation

$$
\widetilde{E}=E^{*} \prod_{l \leq N} 1_{\left\{\left|w_{i_{l}}\left(t_{l}\right)-w_{j_{l}}\left(t_{l}\right)+z_{l}\right| \leq 1\right\}}
$$

Let a minimal graph $\boldsymbol{G}^{\prime}$ be given. We call the vertex $\boldsymbol{x}_{j_{N}}$, the origin of the graph and denote it by $\boldsymbol{v}_{0}$. For any $v \in V\left(G^{\prime}\right)$ denote by $l(v)$ the minimal number of edges connecting $v$ with $v_{0}$; so $l\left(v_{0}\right)=0$. We call $l(v)$ the Irvel of the vertex $v$. Note that if $v, v^{\prime} \in E\left(G^{\prime}\right)$, then $\left|l(v)-l\left(v^{\prime}\right)\right|=1$.

Now we construct the graph $G^{\prime}$ for which the statement of the lemma is valid. Let

$$
\left(\left(i_{1}, \ldots, i_{N}\right),\left(j_{1}, \ldots, j_{N}\right)\right) \in(I J)_{N, m}^{k}, \operatorname{Card} \mathcal{J}=r .
$$

Denote by $G^{\prime}=G\left(i_{1}, \ldots, i_{N} ; j_{1}, \ldots, j_{N}\right)$ the minimal graph with the edges $\left\{\left\{\mathrm{x}_{i_{l_{u}}}, x_{j_{l_{u}}}\right\}\right.$, $u=1, \ldots r\}$ where

$$
l_{u}=\max \left\{l<N: \operatorname{Card}\left\{j_{l}, \ldots, j_{N}\right\} \cap[m+1, n]=r+1-u\right\} .
$$

It is easy to see that the inequality is valid 


$$
\left.\widetilde{E} \leq E^{*} \prod_{l \in \Lambda\left(G^{\prime}\right)} 1_{\left\{\left|\tilde{x}_{i_{l}}^{*}\left(t_{l}\right)-\tilde{x}_{j_{l}}^{*}\left(t_{l}\right)\right| \leq 1\right\}}\right)^{\frac{1}{2}}\left(\widetilde{E}_{2}\left(G^{\prime}\right)\right)^{\frac{1}{2}}
$$

where

$$
\Lambda\left(G^{\prime}\right)-\left\{l<N: i_{l}, j_{l} \in \mathcal{J},\left\{x_{i_{l}}, x_{j_{l}}\right\} \in E\left(G^{\prime}\right)\right\}, \Lambda^{\prime}\left(G^{\prime}\right)=[1, N] \backslash \Lambda\left(G^{\prime}\right) .
$$

Denote

$$
\Lambda^{v}\left(G^{\prime}\right)=\left\{l<N: i_{l}, j_{l} \in \mathcal{J},\left\{x_{i_{l}}, x_{j_{l}}\right\} \in E\left(G^{\prime}\right), v\left(x_{i_{l}}\right) \vee v\left(x_{j_{l}}\right)=v\right\},
$$

and

$$
\Lambda_{0}\left(G^{\prime}\right)=\bigcup_{k \geq 0} \Lambda^{2 k}\left(G^{\prime}\right), \Lambda_{1}\left(G^{\prime}\right)=\bigcup_{k \geq 0} \Lambda^{2 k+1}\left(G^{\prime}\right)
$$

Introduce the r.v.

$$
\xi_{l}=1_{\left\{\left|\widetilde{x}_{i l}^{*}\left(t_{l}\right)-\widetilde{x}_{j_{l}}^{*}\left(t_{l}\right)\right| \leq 1\right.}, l<N, \xi_{N}=1_{\left\{\left|w_{j_{N}}\left(t_{N}\right)+z_{N}\right| \leq 1\right\}} .
$$

It is easy to see that the sets of r.v. $\left\{\xi_{l}, l \in \Lambda^{2 k}\right\}\left(G^{\prime}\right)$ are independent for different $k$ as well as the sets $\left\{\xi_{l}, l \in \Lambda^{2 k+1}\left(G^{\prime}\right)\right.$. Using the Cauchy inequality we can write

$$
\begin{gathered}
\widetilde{E} \leq\left(E^{*} \xi_{N} \prod_{l \in \Lambda_{0}\left(G^{\prime}\right)} \xi_{l}\right)^{1 / 2}\left(E^{*} \prod_{l \in \Lambda_{1}\left(G^{\prime}\right)} \xi_{l}\right)^{1 / 2} \\
=\left(E^{*} \xi_{N} \prod_{r \geq 1} E^{*} \prod_{l \in \Lambda^{r}\left(G^{\prime}\right)} \xi_{l}\right)^{1 / 2}
\end{gathered}
$$

Note that

$$
\Lambda^{v}\left(G^{\prime}\right)=\bigcup_{k} \Lambda_{k}^{v}\left(G^{\prime}\right)
$$

where the sets $\Lambda_{k}^{v}\left(G^{\prime}\right)$ are determined by the sets

$$
X_{k}^{v}=\bigcup_{l \in \Lambda_{k}^{r}\left(G^{\prime}\right)}\left\{x_{i_{l}}, x_{j_{l}}\right\}
$$

which are determined by their properties;

a) $\bigcup_{k, v} X_{k}^{v}=X(\mathcal{J})$,

b) $\quad X_{k}^{v} \bigcap X_{k^{\prime}}^{v^{\prime}}=\emptyset$ if $\left|k-k^{\prime}\right|+\left|v-v^{\prime}\right|>0$,

c) $\operatorname{card} X_{k}^{v}>1, v-1 \leq l(x) \leq v$ if $x \in X_{k}^{v}$,

d) the set $X_{k}^{v}$ contains only one element $z$ for which the relation $l(z)=v-1$ is fulfilled.

Property b) implies the relation

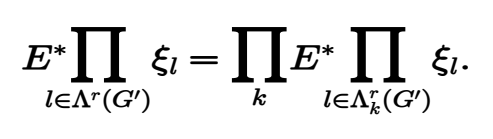


Let $\boldsymbol{w}(t)$ be the Wiener process in $R^{d}$, and $z \in R^{d}$. Then for any $\bar{\theta} \in(\mathbf{0}, \mathbf{1} / \mathbf{2})$, there exists a constant $\bar{c}>1$ for which the inequality is held

$$
E 1_{\{|w(t)-z| \leq 1\}} \leq \bar{c} \exp \left\{-\frac{\bar{\theta}}{t}\left(|z|^{2}-1\right) \vee 0\right\}
$$

So

$$
\begin{aligned}
& E^{*}\left(\prod_{i \leq k}\left(1_{\left\{\left|w_{n_{0}}\left(s_{i}\right)-w_{n_{i}}\left(s_{i}\right)+d_{i}\right| \leq 1\right\}}\right) / w_{n_{0}}(\cdot)\right) \\
\leq & \bar{c}^{k} \exp \left\{-\sum_{i} \frac{\bar{\theta}^{\prime}}{s_{i}}\left(\left|d_{i}+w_{n_{0}}\left(s_{i}\right)\right|^{2}-1\right) \vee 0\right\},
\end{aligned}
$$

where $d_{i}=x_{n_{0}}-x_{n_{i}}$. This implies the inequality

$$
K\left(n_{0}, \ldots, n_{1} ; s_{1}, \ldots, s_{k}\right) \leq c_{3}^{k} \exp \left\{-\sum_{i \leq k} \frac{\theta^{\prime}}{s_{i}}\left(\left|d_{i}\right|^{2}-1\right) \vee 0\right\}
$$

with some $c^{3}>1$ and $\theta^{\prime}>0$. This inequality and the following one

imply the relation

$$
\left|z_{n}\right|^{2}=\left|w_{i_{N}}\left(t_{N}\right)+x_{i_{N}}-x_{j_{N}}\right|^{2} \geq \lambda\left|x_{i_{N}}-x_{j_{N}}\right|^{2}-\frac{\lambda}{1-\lambda}\left|w_{i_{N}}\left(t_{N}\right)\right|^{2}
$$

$$
\widetilde{E} \leq c_{3}^{c a r d} \exp \left\{-\sum_{l \in \Lambda\left(G^{\prime}\right)} \frac{\theta}{t_{1}}\left(\left|x_{i_{l}}-x_{j_{l}}\right|^{2}-1\right) \vee 0+\frac{\theta}{t_{N}}\left|w_{i_{N}}\left(t_{N}\right)\right|^{2}\right\}\left(\widetilde{E}_{2}\left(G^{\prime}\right)\right)^{1 / 2}
$$

with some $\boldsymbol{\theta}>\mathbf{0}$. This formula and formulas (37),(38) imply the proof of the lemma.

\section{The Condition of the Quasistability of Gradient Systems}

Consider the graph $\left.G^{\prime}\left(i_{1}, \ldots, i_{N}\right) ; j_{1}, \ldots j_{N}\right)$ introduced in the proof of Lemma 6 . Let $\mathcal{J}=\left\{j_{l}, l \leq N\right\} \backslash[1, m]$, and $r=\operatorname{card} \mathcal{J}$.

Set $z_{k}=x_{l_{j_{k}}}, k=1, \ldots r$. Denote by $\operatorname{MOG}\left(z_{1}, \ldots, z_{r}\right)$ the set of minimal ordered graphs $G$ with $V(G)=Z$ and the origin $z_{r}$. Let $P_{z_{r}}$ be the set of invertible mapping $U: Z \rightarrow Z$ for which $U\left(z_{r}\right)=z_{r}$. For $G^{\prime}, G^{\prime \prime} \in M O G\left(Z ; z_{r}\right)$ we write $G^{\prime} \sim G^{\prime \prime}$ if the relation $G^{\prime \prime}=U\left(G^{\prime}\right)$ is fulfilled for some $U \in \mathcal{P}_{z_{r}}$. For $G^{\prime} \in M O G\left(Z ; z_{r}\right)$, we set

$$
\nu\left(G^{\prime}\right)=\operatorname{card}\left\{U\left(G^{\prime}\right): U \in \mathcal{P}_{z_{r}} .\right.
$$

It is evident that $\nu\left(G^{\prime}\right)=\nu\left(G^{\prime \prime}\right)$ if $G^{\prime} \sim G^{\prime \prime}$.

Introduce the functions

$$
W_{r}\left(G^{\prime} ; \lambda, \bar{x}, z_{1}, \ldots, z_{r}\right)
$$




$$
\left.=\exp \left\{-\lambda\left(\left|\bar{x}-z_{r}\right|^{2}+\sum\left|z_{i}-z_{j}\right|^{2} \mathbf{1}_{\left\{\left\{z_{i}, z_{l}\right\} \in E\left(G^{\prime}\right)\right\}}\right)\right\} \prod_{i<j} \mathbf{1}_{z_{i} \neq z_{j}}\right\} .
$$

Lemma 7: Let a sequence of ordered graphs $\left\{G_{i}^{\prime}, i \leq L\right\}$ from $M O G\left(z_{1}, \ldots, z_{r}\right)$ satisfy the conditions

a) $G_{i}^{\prime} \nsim G_{j}^{\prime}$ if $i<j$,

b) for any graph $G^{\prime} \in \operatorname{MOG}\left(z_{1}, \ldots, z_{r}\right)$, the relation is true

$$
\sum_{i \leq L} 1_{\left\{G^{\prime} \sim G_{i}^{\prime}\right\}}=1
$$

Let $\gamma_{0}^{n}$ be the configuration with the support $\left\{x_{k}: k \in[m+1, n]\right\}$. Then the inequality is held

$$
\begin{gathered}
\sum_{i_{l}, j_{l}, l \in \Lambda\left(G^{\prime}\right)}\left|B^{k}\left(N ; i_{1}, \ldots, i_{N} ; j_{1}, \ldots, j_{N} ; t_{1}, \ldots, t_{N}\right)\right| \leq \bar{c}^{r} \prod\left(t_{1}, \ldots, t_{N}\right) \\
\sum_{i \leq L} \int_{X(\mathcal{J})} \ldots \int_{X(\mathcal{J})}^{\left(\sup ^{\prime} \in W_{r}\left(G_{i}^{\prime}, \theta / t, w_{k}\left(t_{N}\right), y_{1}, \ldots, y_{r}\right) \gamma_{0}^{n}\left(d y_{1}\right) \ldots \gamma_{0}^{n}\left(d y_{r}\right)\right.}
\end{gathered}
$$

The proof follows from Lemma 6, and the formula

$$
\begin{gathered}
\sum_{i_{l}, j_{l}, l \in \mathcal{J}\left(G^{\prime}\right)}\left|B_{m, n}^{k}\left(N ; i_{1}, \ldots, i_{N} ; j_{1}, \ldots, j_{N} ; t_{1}, \ldots, t_{N}\right)\right| \\
=\sum_{i \leq L} \sum\left|B_{m, n}^{k}\left(N ; i_{1}, \ldots, i_{N} ; j_{1}, \ldots, j_{N} ; t_{1}, \ldots, t_{N}\right)\right| 1_{\left\{G\left(i_{1}, \ldots, i_{N} ; j_{1}, \ldots, j_{N}\right) \sim G_{i}^{\prime}\right\}} .
\end{gathered}
$$

We use the notation

$$
\begin{gathered}
A_{r}\left(\lambda, \bar{x}, z_{1}, \ldots, z_{r}\right)=\sup \left\{\sum_{i \leq L} W_{r}\left(G_{i}^{\prime}\left(\lambda, \bar{x}, U z_{1}, \ldots, U z_{r}\right): U \in \mathcal{P}_{z_{r}}\right\}\right. \\
A_{r}(\lambda, \bar{x}, Z)=\int_{Z} \ldots \int_{Z} A_{r}\left(\lambda, \bar{x}, z_{1}, \ldots, z_{r}\right) \gamma_{0}\left(d z_{1}\right) \ldots \gamma_{0}\left(d z_{r}\right)
\end{gathered}
$$

where $Z \subset S_{\gamma_{0}}, \operatorname{Card} Z=r$.

Theorem 1: Assume the value $\boldsymbol{\alpha}$ in condition $(\mathrm{P})$ satisfies the inequality $\boldsymbol{\alpha}<\mathbf{1}$, and the initial configuration $\gamma_{0} \in \Gamma$ for a gradient system satisfy condition (EGFPS) and the conditions: fulfilled

1) for any $\boldsymbol{\lambda}>0$ there exists constant $\boldsymbol{a}(\boldsymbol{\lambda})>0$ for which the inequalities are

$$
\int \exp \left\{-\lambda(x-y)^{2}\right\}(\log (1+|y|))^{k} \gamma_{0}(d y)
$$




$$
<(a(\lambda))^{k}\left((\log (k+1))^{k+1}+(\log (1+|y|))^{k+1}\right),
$$

2) Denote

$$
A_{N}(\lambda)=\sup \left\{\sum_{\bar{x} \in Z} A_{r}(\lambda, \bar{x}, Z): Z \subset\left\{x_{1} ; \ldots ; x_{N}\right\}, r \leq N\right\}
$$

then for all $\boldsymbol{\lambda}>\mathbf{0}$ the relation is valid

$$
\lim _{N} \frac{\log A_{N}(\lambda)}{\log N}=0
$$

Under these conditions the gradient system is quasistable.

Proof: It suffices to prove the existence of a limit in probability

$$
\lim _{n} \rho_{n, m}(t)
$$

and the relation

for all $t>0$.

$$
E \lim _{n} \rho_{n, m}(t)=1
$$

Note that for any sequences

$$
\left\{\left\{i_{1}, \ldots, i_{N}\right\} ;\left\{j_{1}, \ldots, j_{N}\right\}\right\} \in[m+1, \infty)^{N} \times[1, \infty)^{N}
$$

there exists a limit in probability

$$
\lim _{n} B_{m, n}^{k}\left(N ; i_{1}, \ldots, i_{N} ; j_{1}, \ldots, j_{N}, t_{1}, \ldots, t_{N}\right)
$$

which we denote by

$$
B_{m}^{k}\left(N ; i_{1}, \ldots, i_{N} ; j_{1}, \ldots, j_{N} ; t_{1}, \ldots, t_{N}\right),
$$

represented by the right part of equality (35). Introduce the stochastic process in $\left(R^{d}\right)^{m}$

$$
\widetilde{X}^{*}(t)=\left(\widetilde{x}_{1}^{*}(t) ; \ldots ; \widetilde{x}_{m}^{*}(t)\right)
$$

and functions

by the relations

$$
\Phi_{r}^{k}(m, n, N ; \cdot, \ldots, \cdot), \Phi_{r}^{k}(m, N ; \cdot, \ldots, \cdot):\left(\left(\left(R^{d}\right)^{m}\right)^{N} \rightarrow R^{d}\right.
$$

$$
\begin{gathered}
\Phi_{r}^{k}\left(m, n, N ; \widetilde{X}_{m}^{*}\left(t_{1}\right), \ldots, \widetilde{X}_{m}^{*}\left(t_{N}\right)\right) \\
=\sum_{\operatorname{card}\left\{j_{1}, \ldots, j_{N}\right\} \backslash[1, m]=r} B_{m, n}^{k}\left(N ; i_{1}, \ldots, i_{N} ; j_{1}, \ldots, j_{N} ; t_{1}, \ldots, t_{N}\right), \\
\Phi_{r}^{k}\left(m, N ; \widetilde{X}_{m}^{*}\left(t_{1}\right), \ldots, \widetilde{X}_{m}^{*}\left(t_{N}\right)\right)
\end{gathered}
$$




$$
=\sum_{\operatorname{card}\left\{j_{1}, \ldots, j_{N}\right\} \backslash[1, m]=r} B_{m}^{k}\left(N ; i_{1}, \ldots, i_{N} ; j_{1}, \ldots, j_{N} ; t_{1}, \ldots, t_{N}\right)
$$

It follows from Lemma 7 that

$$
\begin{gathered}
\left|\Phi_{r}^{k}\left(m, n, N ; \widetilde{X}_{m}^{*}\left(t_{1}\right), \ldots, \widetilde{X}_{m}^{*}\left(t_{N}\right)\right)\right| \vee\left|\Phi_{r}^{k}\left(m, N ; \widetilde{X}_{m}^{*}\left(t_{1}\right), \ldots, \widetilde{X}_{m}^{*}\left(t_{N}\right)\right)\right| \\
\leq \bar{c}^{r} \Pi_{N}\left(t_{1}, \ldots, t_{N}\right)\left(\sup _{G^{\prime} \in M O G\left(Z, z_{r}\right)} \widetilde{E}_{2}\right)^{1 / 2} \exp \left\{\left(\theta / t_{N}\right)\left(\left|w_{k}\left(t_{N}\right)\right|^{2}-1\right) \vee 0\right\}(45) \\
\sum_{i \leq L} \int \ldots \int W_{r}\left(G_{i}^{\prime}, \theta / t, w_{k}\left(t_{N}\right), y_{1}, \ldots, y_{r}\right) \gamma_{0}\left(d y_{1}\right) \ldots \gamma_{0}\left(d y_{r}\right) .
\end{gathered}
$$

It follows from Lemma 5 that

$b_{m, n}^{k}(t)=\sum_{N \geq 1} \sum_{r \leq n-m} \int \ldots \int_{0<t_{1}<\ldots<t_{N}<t} \Phi_{r}^{k}\left(m, n, N ; \widetilde{X}_{m}^{*}\left(t_{1}\right), \ldots, \widetilde{X}_{m}^{*}\left(t_{N}\right)\right) d t_{1} \ldots d t_{N}$.

Set

$$
b_{m}^{k}(t)=\sum_{N \geq 1} \sum_{r \leq n-m} \int \ldots \int_{0<t_{1}<\ldots<t_{N}<t} \Phi_{r}^{k}\left(m, N ; \widetilde{X}_{m}^{*}\left(t_{1}\right), \ldots, \widetilde{X}_{m}^{*}\left(t_{N}\right)\right) d t_{1} \ldots d t_{N}
$$

Investigate the convergence of the series in formula (47). First we note that for some $\boldsymbol{\beta}>\mathbf{0}$ and $\boldsymbol{C}>0$, the inequality is valid

$$
\int \ldots \int_{0<t_{1}<\ldots<t_{N}<t} \Pi_{N}\left(t_{1}, \ldots, t_{N}\right) d t_{1}, \ldots, t_{N} \leq C t^{N}(\Gamma(\beta N))^{-1}
$$

Now we obtain a proper estimation for $\widetilde{E}_{2}$. Note that it depends on $\left\{\left(i_{l} ; j_{l}\right), l \in \Lambda^{\prime}\left(G^{\prime}\right)\right\}$, so we will write

$$
\widetilde{E}_{2}\left(G^{\prime}\right)=\widetilde{E}_{2}\left(G^{\prime} ; i_{1}, \ldots, i_{N} ; j_{1}, \ldots, j_{N}\right),
$$

the function in the right-hand side of the equality depends only of those $\left(i_{l} ; j_{l}\right)$ for which $l \in \Lambda^{\prime}\left(G^{\prime}\right)$. Introduce the subsets of the set $\Lambda^{\prime}\left(G^{\prime}\right):\left\{\Lambda_{i}, i \leq s\right\}$ satisfying the conditions

$$
\bigcup_{i \leq s} \Lambda_{i}=\Lambda^{\prime}\left(G^{\prime}\right), \bigcup_{i<j} \Lambda_{i} \bigcap \Lambda_{j}=\emptyset, \operatorname{Card} \Lambda_{i}=r, i<s, \operatorname{Card} \Lambda_{s} \leq r
$$

and $\min \boldsymbol{\Lambda}_{i}>\max \boldsymbol{\Lambda}_{i+1}$. Set

$$
\eta_{i}=\prod_{l \in \Lambda_{i}} 1_{\left\{\left|\bar{x}_{i_{l}}^{*}\left(t_{l}\right)-\bar{x}_{j_{l}}^{*}\left(t_{l}\right)\right| \leq 1\right\}}
$$

For some $C_{1}>0, \delta>0$ we can write the inequality 


$$
\widetilde{E}_{2}\left(G^{\prime}\right) \leq C_{1}^{N} \prod_{i \leq s}\left(E^{*} \eta_{i}\right)^{\delta}
$$

Let $G^{\prime}$ be a minimal ordered graph with the vertices $\left\{y_{1}, \ldots, y_{r}\right\}$ and the origin $y_{r}$. It follows from condition 1) of the theorem that

$$
\int \ldots \int W_{r}\left(G^{\prime}, \lambda, \bar{x}, y_{1}, \ldots, y_{r}\right) \gamma_{0}\left(d y_{1}\right) \ldots \gamma_{0}\left(d y_{r}\right) \leq(a(\lambda))^{r} \Delta_{r}(\bar{x})^{r+1}
$$

where

$$
\Delta_{r}(\bar{x})=\log (1+r)+\log (1+|\bar{x}|) .
$$

Using Lemmas 6 and 7 for the estimation of $\boldsymbol{E}^{*} \boldsymbol{\eta}_{i}$ we can obtain the inequality

$$
\begin{gathered}
\sum_{i \leq L} \sum \widetilde{E}_{2}\left(G_{i}^{\prime} ; i_{1}, \ldots, i_{N} ; j_{1}, \ldots, j_{N}\right) 1_{\left\{\left(i_{1}, \ldots, i_{N}\right),\left(j_{1}, \ldots, j_{N}\right) \in(I J)_{N, m}^{k}, \operatorname{Card} \mathcal{J}\right\}} \\
\leq\left(A_{N}\left(\frac{\delta \theta}{2 t}\right)\right)^{s} .
\end{gathered}
$$

These inequalities and formula (45) imply the inequality

$$
\begin{aligned}
\int \ldots & \int_{0<t_{1}<\ldots<t_{n}} \mid \Phi_{r}^{k}\left(m, N: \widetilde{X}_{m}^{*}\left(t_{1}\right), \ldots, \widetilde{X}_{m}^{*}\left(t_{N}\right) \mid d t_{1} \ldots d t_{N} \leq L(\Gamma(\beta N))^{-1}\right. \\
& \bar{c}_{1}^{r}\left(A _ { N } ( \frac { \delta \theta } { 2 t } ) ^ { r } \left(\sup _{s \leq t} \Delta_{r}\left(\widetilde{x}_{k}^{*}(s)\right)^{r+1} \sup _{s \leq t} \exp \left\{(\theta / s)\left(\left|w_{k}(s)\right|^{2}-1\right) \vee 0\right\}\right.\right.
\end{aligned}
$$

where $\overline{\boldsymbol{c}}_{1}$ is a constant, and $\boldsymbol{L}$ was introduced in Lemma 7. It can be shown that

$$
\lim _{r \rightarrow \infty}(\log L / r \log r=0)
$$

This implies the local uniform convergence in $t$ of the series in formula (47).

This implies the continuity of the functions $\widehat{b}_{m}^{k}(r, t)$ for all $r$ and continuity of the function $b_{m}^{k}(t)$. Introduce the function

$$
\widehat{\rho}(t)=\exp \left\{\sum_{k \leq m} \int_{0}^{t} b_{m}^{k}(s) d w_{k}(s)-\frac{1}{2} \int_{0}^{t}\left|b_{m}^{k}(s)\right|^{2} d s\right\} .
$$

Inequality (50) implies the relation

$$
\rho_{m, n}(t) \rightarrow \widehat{\rho}(t)
$$

in probability as $n \rightarrow \infty$. Introduce the stopping time

$$
\tau_{T}=\inf \left\{t: \sum_{k \leq m} \int_{0}^{t}\left|b_{m}^{k}(s)\right|^{2} d s>T\right\}
$$


Then the stochastic process $\widehat{\rho}\left(\boldsymbol{t} \wedge \tau_{T}\right)$ is a martingale, and

$$
E \widehat{\rho}\left(t \wedge \tau_{T}\right)=1
$$

The proof of the theorem is a consequence of the relation

$$
P\left\{\lim _{T \rightarrow \infty} \tau_{T}=\infty\right\}=1 .
$$

\subsection{The Condition of Quasistability of Hamiltonian Systems}

Let $\left\{x_{k}^{n}(t), k \leq n\right\}$ be the solution to system (2) with the initial condition

$$
x_{k}^{n}(0)+x_{k} \in R^{d}, \dot{x}_{k}^{n}(0)=v_{k} \in R^{d},
$$

where $\left\{x_{k}, k \leq n\right\}$ is the set of different points from $R^{d}$.

Denote by $\rho_{n}(t)$ the density of the distribution of the stochastic process

$$
\left\{X_{n}(s), s \leq t\right\}, X_{n}(s)=\left\{x_{k}^{n}(s), k \leq n\right\}
$$

with respect to the distribution of the stochastic process

$\left\{X^{*}(s), s \leq t\right\}, X^{*}(s)=\left\{x_{k}+s v_{k}+u_{k}(s), k \leq n\right\}, u_{k}(s)=\int_{0}^{s} w_{k}(t) d t, k=1,2, \ldots$

This density is determined by formula (23).

Let $m<n$, set

$$
\rho_{n, m}(t)=E\left(\rho_{n}(t) / \mathcal{F}_{t}^{m}\right) .
$$

Lemma 8: a) The formula is fulfilled

$$
\rho_{n, m}(t)=\exp \left\{\sum_{k \leq m}\left(\int_{0}^{t}\left(\tilde{b}_{n, m}^{k}(s), d w_{k}(s)\right)-\frac{1}{2} \int_{0}^{t}\left|\tilde{b}_{n, m}^{k}(s)\right|{ }^{2} d s\right\}\right.
$$

where the function $\widetilde{\boldsymbol{b}}_{n, m}^{k}(\boldsymbol{t})$ is determined by formula (33) with the functions $\widetilde{\boldsymbol{B}}_{n, m}^{k}(\cdot)$ instead of the functions $\boldsymbol{B}_{n, m}^{k}$, and the function $\widetilde{\boldsymbol{B}}_{n, m}^{k}(\cdot)$ are determined by formula (34) with the functions $\left\{x_{i}^{*}(t), i \leq n\right\}$ instead of the functions $\left\{\widetilde{x}_{i}^{*}(t), i \leq n\right\}$.

b) Let

$$
\left\{\left\{i_{1}, \ldots, i_{N}\right\} ;\left\{j_{1}, \ldots, j_{N}\right\}\right\} \in(I J)_{N}^{k},
$$

where the set $(\boldsymbol{I J})_{N}^{k}$ is determined by Lemma 5 , and the functions

$$
\widetilde{B}_{n, m}^{k}\left(N ; i_{1}, \ldots, i_{N} ; j_{1}, \ldots, j_{N} ; t_{1}, \ldots, t_{N}\right)
$$


are determined by formula (35) with $\left\{\widetilde{x}_{i}^{*}(t), i \leq n\right\}$ instead of $\left\{x_{i}^{*}(t), i \leq n\right\}$. Then there exist some constants $\boldsymbol{c}>0, \boldsymbol{\theta}>0$, and a graph $G^{\prime} \in M G(\mathcal{J})$ for which the inequality is fulfilled

$$
\begin{gathered}
\left|\widetilde{B}_{n, m}^{k}\left(N ; i_{1}, \ldots, i_{N} ; j_{1}, \ldots, j_{N} ; t_{1}, \ldots, t_{N}\right)\right| \leq \Pi_{N}\left(i_{1}, \ldots, i_{N} ;\left(\tilde{E}_{2}\left(G^{\prime}\right)\right)^{1 / 2}\right. \\
W_{r}\left(G^{\prime}, \theta / t^{3}, x_{k}^{*}\left(t_{N}\right), z_{1}, \ldots, z_{r}\right),
\end{gathered}
$$

where $\boldsymbol{\Pi}_{N}$ and $\tilde{\boldsymbol{E}}_{2}$ where introduced in Lemma $6,\left\{z_{1}, \ldots, z_{r}\right\}$ are the vertices of the graph $\boldsymbol{G}^{\prime}$, and the function $\boldsymbol{W}_{r}$ were introduced before Lemma 7 .

The proof can be performed in the same way as for Lemmas 5 and 6 .

Introduce the function

$$
\rho(t, R)=\inf \left\{\frac{\left|x_{i}-x_{j}+s\left(v_{i}-v_{j}\right)\right|}{\left|x_{i}-x_{j}\right|}: s \leq t,\left|x_{i}\right| \leq R,\left|x_{j}\right| \leq r, i \neq j\right\}, t>0, R>0 .
$$

Theorem 2: Let $\alpha<1$ and the initial condition $\widetilde{\gamma}_{0} \in \Gamma^{2}$ of a Hamiltonian system satisfy condition $\rho(t, R)=O(\log R)$ for all $t>0$. Let the configuration $\gamma_{0} \in \Gamma$ with support $S_{\gamma_{0}}=\left\{x_{k}, k \geq 1\right\}$ satisfies conditions 1$\left.), 2\right)$ of Theorem 1. Then the Hamiltonian system is quasistable.

The proof is very similar to the proof of Theorem 1.

\section{Acknowledgment}

I would like to recognize Professor Yu. Kondratiev with whom I had many fruitful discussions on the quasistability theme. I also had useful discussions with Professors R. Khasminskii, N. Krylov, and M. Portenko. I am very grateful to all of them.

\section{References}

[1] Fritz, J., Gradient dynamics of infinite point systems, Annals of Prob. 15:4 (1987), 78514.

[2] Girsanov, I.V., On a transformation of some class of stochastic processes by absolute continuous changing of measures, Theory of Prob. and Applic. 5 (1960), 314-330.

[3] Skorokhod, A.V., On differentiability of measures corresponding to stochastic processes II: Markov processes, Theory of Prob. and Applic. 5 (1960), 49-53.

[4] Skorokhod, A.V., Stochastic Equations for Complex Systems, Riedel 1988.

[5] Skorokhod, A.V., On the regularity of many particle dynamical systems perturbed by white noise, J. Appl. Math. and Stoch. Anal. 9 (1996), 427-437.

[6] Skorokhod, A.V., On infinite systems of stochastic differential equations, JH. Methods of Funct. Anal. and Topol. 4 (1999), 54-61. 


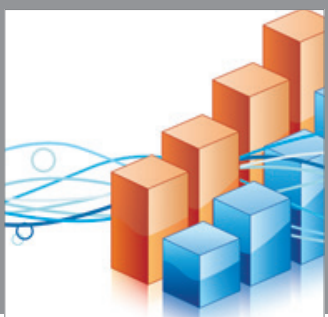

Advances in

Operations Research

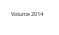

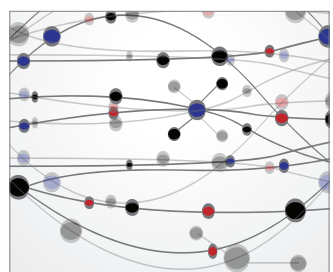

\section{The Scientific} World Journal
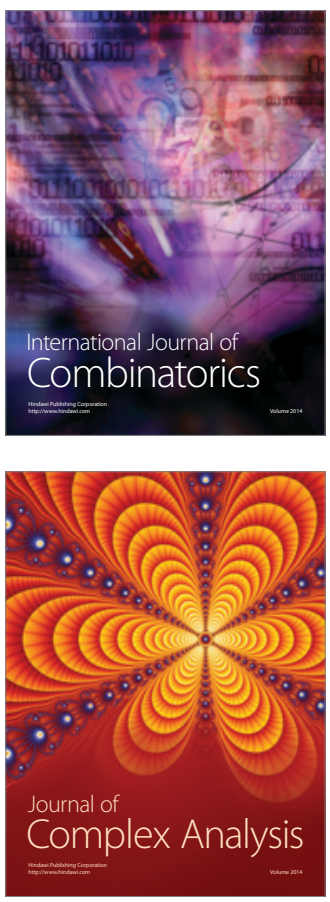

International Journal of

Mathematics and

Mathematical

Sciences
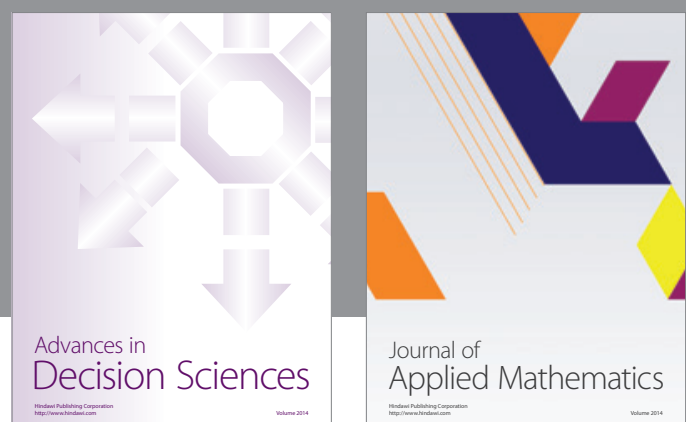

Journal of

Applied Mathematics
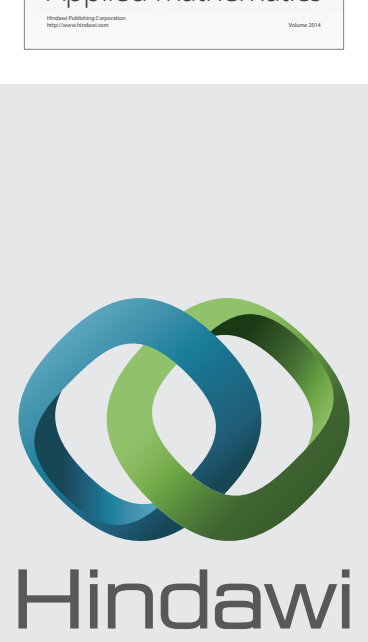

Submit your manuscripts at http://www.hindawi.com
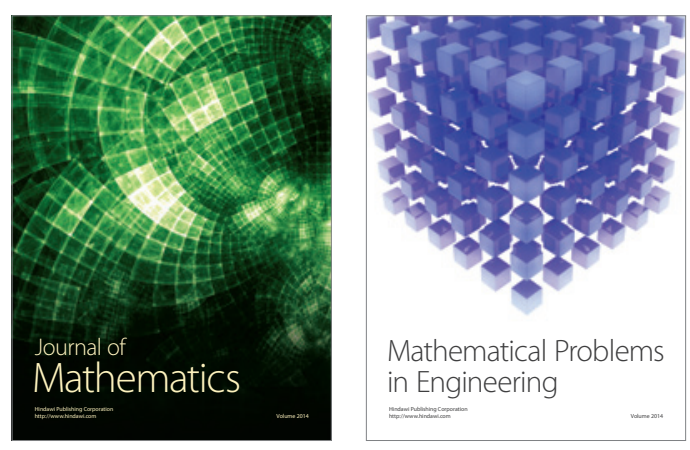

Mathematical Problems in Engineering
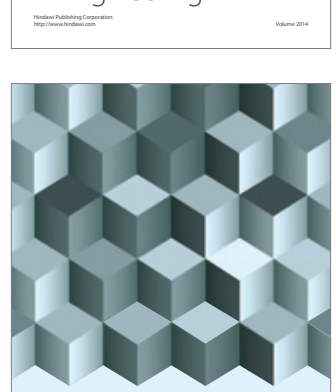

Journal of

Function Spaces
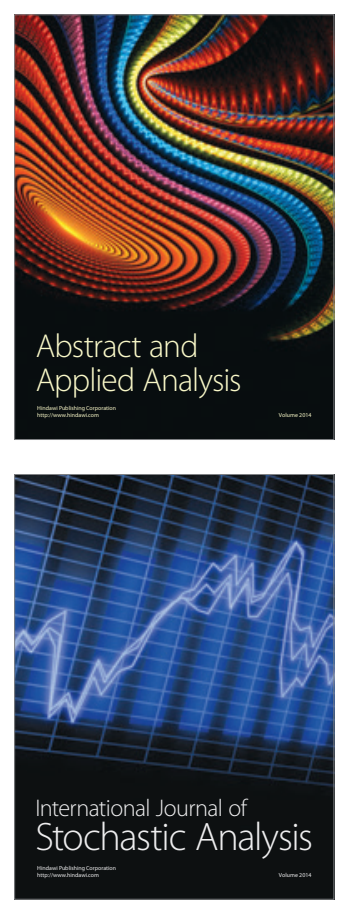

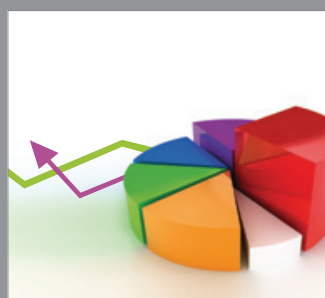

ournal of

Probability and Statistics

Promensencen
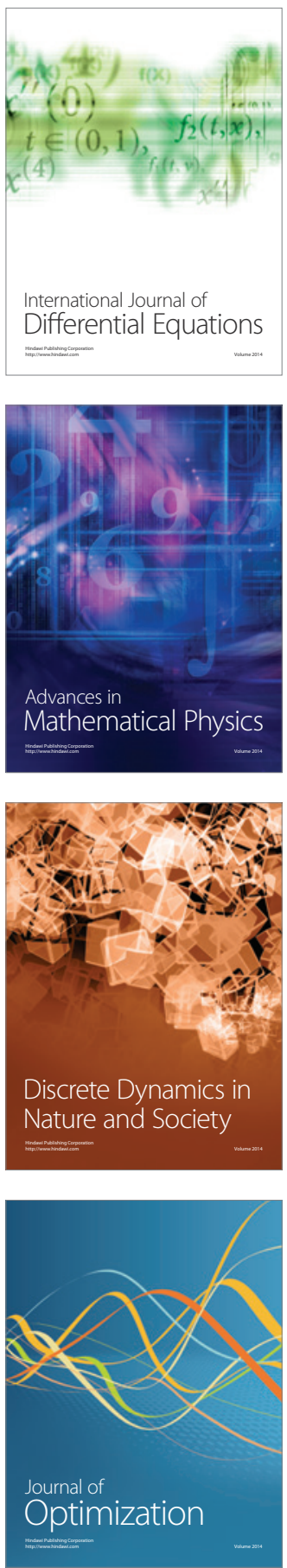